\title{
Palliativdienste in den deutschen onkologischen Spitzenzentren
}

Palliative Care Teams in the German Comprehensive

\section{Cancer Centers}

Autoren

Susanne Gahr ${ }^{1}$, Julia Berendt ${ }^{2}$, Sarah Lödel ${ }^{1}$, Christoph Ostgathe ${ }^{1}$, Steffen T. Simon ${ }^{3}$, Mitra Tewes ${ }^{4}$, Kirsten Zader ${ }^{5,6}$, Jacqueline Schwartz ${ }^{5}$, Martin Neukirchen ${ }^{5,6}$

\section{(ㄷ) (1) $(9)$}

Institute

1 Palliativmedizinische Abteilung, Comprehensive Cancer Center CCC Erlangen-EMN, Universitätsklinikum Erlangen, Friedrich-Alexander-Universität Erlangen-Nürnberg

2 Landesamt für Gesundheit und Lebensmittelsicherheit, Nürnberg

3 Zentrum für Palliativmedizin, Centrum für Integrierte Onkologie Aachen/Bonn/Köln/Düsseldorf, Universität zu Köln und Uniklinik Köln

4 Westdeutsches Tumorzentrum - Comprehensive Cancer Center, Innere Klinik (Tumorforschung), Universitätsklinikum Essen

5 Interdisziplinäres Zentrum für Palliativmedizin, Centrum für Integrierte Onkologie Aachen/Bonn/Köln/Düsseldorf, Universitätsklinikum Düsseldorf, Heinrich Heine Universität Düsseldorf

6 Klinik für Anästhesiologie, Universitätsklinikum Düsseldorf, Heinrich Heine Universität Düsseldorf

Schlüsselwörter

Palliativmedizinscher Dienst, Comprehensive Cancer Center, Multiprofessionalität, Zusatzentgelt, palliativmedizinische Komplexbehandlung

\section{Key words}

palliative care team, Comprehensive Cancer Center, multi-professionality, additional charge, complex palliative medical care

\section{Bibliografie}

DOI https://doi.org/10.1055/a-0961-7236

Online-Publikation: 27.8.2019

Dtsch Med Wochenschr 2019; 144: e153-e159

(c) Georg Thieme Verlag KG, Stuttgart · New York ISSN 0012-0472

\section{Korrespondenzadresse}

PD Dr. Susanne Gahr

Universitätsklinikum Erlangen, Comprehensive Cancer Center EMN-Erlangen, Friedrich-Alexander-Universität Erlangen-

Nürnberg (FAU), Palliativmedizinische Abteilung,

Krankenhausstraße 12, 91054 Erlangen

ccc-koordination.pm@uk-erlangen.de

\section{ZUSAMMENFASSUNG}

Hintergrund Ein Palliativdienst ist in der Beratung und Versorgung von Patienten mit einer Tumorerkrankung als Qualitätsindikator anerkannt und in National Cancer Institutes, Vorbild der deutschen Comprehensive Cancer Center (CCC), flächendeckend (92\%) umgesetzt. Daher stellt sich die Frage, wie die Palliativdienste aktuell in die CCCs integriert sind.

Methode Durch eine papierbasierte quantitative Befragung an 16 Standorten der bisher geförderten CCCs wurden von Juli bis August 2017 Angaben zum Vorhandensein, zur personellen Situation, Inanspruchnahme und zu Perspektiven eines Palliativdienstes erfasst. Die Befragung richtete sich an die Leiter der palliativmedizinischen Einheiten der CCCs. Die Daten wurden in SPSS ausgewertet (Häufigkeit, Median, Mittelwert, Spannweite).

Ergebnisse 15 CCCs haben an der Befragung teilgenommen (Rücklauf 94\%). 13 der 15 CCCs verfügen über einen Dienst, der Palliativpatienten mitbehandelt. In 12 von 13 CCCs sind diese in den Regelarbeitszeiten (wochentags 8-16 Uhr) erreichbar. Alle Dienste sind ärztlich besetzt, 11 zusätzlich pflegerisch. 7 Dienste sind neben Arzt und Pflege mit einer weiteren Profession besetzt. Es wurden im Jahr 2016 im Median 4482 Mitbehandlungen durch die Dienste erbracht, davon $80 \%$ als Zusatzentgelte (ZE) ohne Erlös und ohne kodierbaren OPS. Im Jahr 2017 planen 5 Zentren das ZE 2017-133, 2 Zentren das ZE 60 und 3 Zentren beide ZE abzurechnen.

Diskussion Dienste für Palliativpatienten sind in den deutschen CCCs regelhaft vorhanden, erfüllen jedoch nur zur Hälfte die in der S3-Leitlinie geforderte Bedingung der Multiprofessionalität. Das im Jahr 2017 neu eingeführte, stundengenau abrechenbare Zusatzentgelt könnte hier Verbesserungen schaffen und zur Kostendeckung beitragen. Dadurch können entgegen der bisherigen Regelung notwendige und sinnvolle Leistungen mit einer Behandlungsdauer auch unter 7 Tagen abgerechnet werden.

\section{ABSTRACT}

Background A palliative care team is recognized as a quality indicator in the consultation and care of patients with a tumor disease and is used nationally (92\%) in the National Cancer Institutes, model of the German Comprehensive Cancer Center (CCC). This begs the question of how palliative care teams are presently integrated into the CCCs. 
Method From July to August 2017, a paper-based quantitative survey of 16 locations of the CCCs, supported to that date, gathered information on the existence, personnel situation, use and prospects of a specialized inpatient palliative care service. The survey was addressed to the heads of the palliative medical units of the CCCs. The data were evaluated in SPSS (frequency, median, mean, range).

Results Fifteen CCCs took part in the survey (response 94\%). Thirteen of the fifteen CCCs have a service that also treats palliative patients. Twelve of thirteen CCCs of these are attainable during regular working hours (8a. m.-4 p. m. on weekdays). All services are staffed by physicians, additional eleven are staffed by nurses. Seven services are besides physicians and nurses joined by other professions. In 2016, 4482 median co-treatments were provided by the services, $80 \%$ of these as additional charges without revenue and without codable OPS. In 2017, five centers plan to charge ZE 2017-133, two centers ZE 60 and three centers both intend to charge ZE 2017-133.

Conclusions Services for palliative patients exist generally in the German CCCs, but only half of them satisfy the condition of multi-professionality required for the fulfillment of the german guidelines. The new surcharge introduced in 2017, which can be charged on an hourly basis, could create improvements in this regard and contribute to cost recovery. Thus, contrary to the previous arrangement, essential and reasonable performances with a time of treatment of less than seven days can be charged.

\section{Einleitung}

Nach Einrichtung zahlreicher Palliativstationen in Deutschland etablieren sich zunehmend spezialisierte multiprofessionelle Palliativdienste [1]. Diese Palliativdienste stellen eine spezielle Form der stationären Palliativversorgung dar, die außerhalb einer Palliativstation in anderen Fachabteilungen Patienten mit einer nicht heilbaren, lebenslimitierenden Erkrankung und deren Angehörige mitbehandeln und unterstützen [2, 3]. Während eines stationären Aufenthalts soll Patienten mit einer nicht heilbaren Krebserkrankung Kontakt mit einem Palliativdienst angeboten werden [2, 4].

Die im Jahr 2015 erschienene und in 2019 geplante erweiterte und aktualisierte deutsche S3-Leitlinie für Palliativmedizin ordnet eine Palliativstation, eine Tagesklinik und einen Palliativdienst der spezialisierten Palliativmedizin in einem Krankenhaus zu und empfiehlt, dass jedes Krankenhaus, das Patienten mit einer nicht heilbaren Krebserkrankung behandelt, einen Palliativdienst vorhalten soll [2]. Darüber hinaus beschreibt die S3-Leitlinie die Struktur eines Palliativdienstes [2].

Das multiprofessionelle Team eines Palliativdienstes in Deutschland, deren Vorbild die National Cancer Institutes sind [5], sollte als Strukturqualitätskriterium neben Ärzten und Pflegekräften mindestens 1 weitere Profession, wie z. B. psychoonkologische Mitarbeiter, Physiotherapeuten oder Mitarbeiter des Sozialdienstes, umfassen $[2,6]$.

Zur Sicherstellung der Prozessqualität eines in einem CCC ansässigen Palliativdienstes sollte eine Evaluation der Therapieziele und der durchgeführten Behandlungsmaßnahmen gehören $[2,6]$. Regelmäßige Treffen des multiprofessionellen Teams sowie der Austausch mit dem Behandler-Team, wie auch deren Beratung $[2,7,8]$, sollten dies unterstützen. Damit soll eine individuelle Therapieplanung, abgestimmt auf die Symptome und Bedürfnisse des Patienten und seiner Angehörigen, gewährleistet werden.

Der Aufgabenbereich eines spezialisierten Palliativdienstes ist vielfältig und soll neben dem Assessment der Symptome und Bedürfnisse des Patienten und seiner Angehörigen konsekutiv auch die entsprechende symptomorientierte Therapie unter Beachtung des Therapieziels und der Krankheitsbewältigung erfassen
$[2,4]$. Zudem sollen auch eine palliativmedizinisch orientierte Versorgungsplanung und -koordinierung gewährleistet werden [2]. Eine Trauerbegleitung des Patienten und seiner Angehörigen sowie eine Mitbegleitung in der Sterbephase sollen ebenfalls angeboten werden [2].

Palliativdienste sollen im Rahmen einer frühen Integration („Early Integration“) beratend auch in die Versorgung von asymptomatischen Tumorpatienten eingebunden werden. Ziel der „Early Integration“ ist es, bei Diagnosestellung frühzeitig realisierbare Therapieoptionen und -ziele festzulegen. Dadurch bekommen der Patient und seine Angehörigen die Möglichkeit, sich mit der Erkrankung auseinanderzusetzen und sich über Behandlungsoptionen zu informieren. Dabei sollen die individuellen Wünsche und Wertvorstellungen des Patienten berücksichtigt werden [9, 10]. Ein wechselseitiger Austausch zwischen Palliativmedizinern, Onkologen, aber auch Behandlern anderer Fachrichtungen ist für eine optimale palliativmedizinische Versorgung essenziell $[2,6,11]$.

Die Finanzierung der Palliativdienste in Deutschland erfolgt über Zusatzentgelte (ZE) [12].

Erstmals wurde 2005 die Möglichkeit geschaffen, mit dem Operationen- und Prozeduren-Schlüssel (OPS) Code 8-982 („,palliativmedizinische Komplexbehandlung“) auch Leistungen durch einen Palliativdienst in der Palliativmedizin zu erfassen und zu vergüten. Gestaffelt wird über das ZE 60 nach der Behandlungsdauer (OPS-Code 8-982.0: weniger als 7 Tage, OPS-Code 8-982.1: zwischen 7 und 13 Tage, OPS-Code 8-982.2: 14 bis 20 Tage und OPS-Code 8-982.3: ab 21 Tage). Vergütungsrelevant sind nur die Leistungen ab 7 Tagen $[12,13]$.

Im Jahr 2017 wurde infolge des Hospiz- und Palliativgesetzes in Deutschland ein neues Zusatzentgelt ZE 2017-133 mit dem OPSCode 8-98h.0 („spezialisierte palliativmedizinische Komplexbehandlung durch einen internen Palliativdienst“) bzw. Zusatzentgelt ZE 2017-134 mit dem OPS-Code 8-98h.1 („spezialisierte palliativmedizinische Komplexbehandlung durch einen externen Palliativdienst“) eingeführt. Bei dem neuen Zusatzentgelt wird ebenfalls nach Behandlungsdauer gestaffelt, allerdings nicht nach Tagen wie bei dem ZE 60, sondern nach Stunden, die patientennah verbracht werden (von .00 bis unter 2 Stunden bis .0b 55 oder mehr Stunden). Die Höhe der Vergütung des ZE 2017-133 in 
den Jahren 2017 und 2018 wird zunächst auf der Ist-Kostenbasis des Vorjahres berechnet, die durch das Institut für das Entgeltsystem im Krankenhaus (InEK) über die sogenannten InEK-Krankenhäuser ermittelt wird. Bis zum Jahr 2019 gibt es also keine festgelegte Vergütungshöhe und jeder Anbieter muss das Zusatzentgelt individuell mit den Leistungsträgern verhandeln. Die Anpassungen des neuen OPS-Codes anhand der aktuellen Beobachtungen in InEK-Krankenhäusern können somit erst frühestens nach 2 Jahren im DRG-Katalog berücksichtigt werden und spiegeln sich dann erst in der vergütungsfähigen Leistung wider.

Inwieweit sind Palliativdienste in Deutschland überhaupt in der Routineversorgung etabliert? Erfüllen alle Dienste die personellen Mindestvoraussetzungen und wie rechnen diese Palliativdienste ihre Leistungen $a b$ ?

Die folgende Untersuchung soll den Implementierungsstand der multiprofessionellen Palliativdienste in den 16 deutschen, von der Deutschen Krebshilfe geförderten onkologischen Spitzenzentren ermitteln und beschreiben.

\section{Material und Methoden}

Für die Datenerhebung wurde ein Fragebogen mit quantitativen und qualitativen Anteilen entwickelt, der sich an die 16 leitenden Ärzte der palliativmedizinischen Abteilungen, die gleichzeitig Mitglied in der Arbeitsgruppe Palliativmedizin im Netzwerk von der Deutschen Krebshilfe geförderten CCC sind, richtete. Im Juli 2017 wurde der Fragebogen per E-Mail zur manuellen Bearbeitung übermittelt und konnte bis August 2017 per Fax beantwortet werden. Die Fragen bezogen sich auf das Jahr 2016 und wurden anhand der Best-Practice-Empfehlungen zur Integration der spezialisierten Palliativmedizin [6] strukturiert. Neben Fragen zur Etablierung von Palliativdiensten wurden Angaben zur Kooperation der Palliativmedizin mit der Intensivmedizin, zur Implementierung einer palliativmedizinischen Sprechstunde und zum Vorhandensein ärztlicher Rotationsprogramme erhoben. Der Befragungsteil zur Etablierung von Palliativdiensten beinhaltete 2 offene und 6 geschlossene Fragen (von insgesamt 29 Fragen) und wurde anonym ausgewertet. Die Datenerfassung erfolgte mittels IBM SPSS Statistics 21, anschließend wurde eine deskriptive Auswertung der Daten durchgeführt (Häufigkeit, Mittelwert, Median, Spannweite R).

\section{Ergebnisse}

15 der 16 kontaktierten Standorte der CCCs (94\%) (1 CCC umfasst 2 Standorte) nahmen von Juli bis August 2017 an der Datenerhebung teil. 1 CCC-Standort war es im festgelegten Zeitfenster nicht möglich, den Erhebungsbogen zu bearbeiten.

13 von 15 (87\%) der von der Deutschen Krebshilfe geförderten onkologischen Spitzenzentren geben an, über einen Palliativdienst zu verfügen. Diese Angabe wurde unabhängig davon getätigt, ob die in den Best-Practice-Empfehlungen definierten Kriterien für einen Palliativdienst erfüllt werden. 1 Zentrum gibt an, dass der Palliativdienst noch im Aufbau ist. 1 Zentrum hat zum Befragungszeitpunkt nicht ausreichend Personal für einen multiprofessionellen Palliativdienst, weshalb alternativ durch einen Ober-

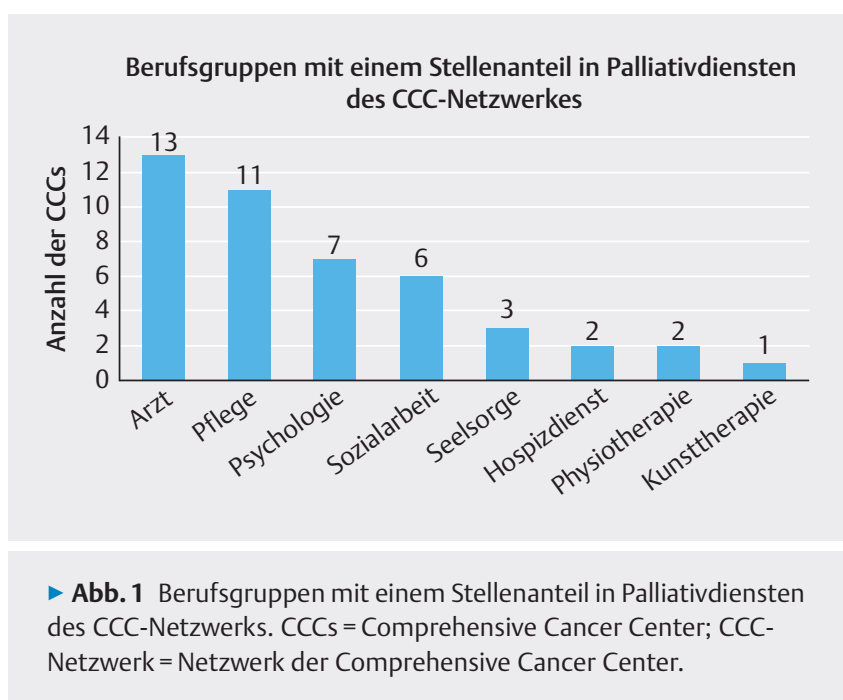

arzt oder eine Oberärztin palliativmedizinische Konsile erbracht werden. In 1 Spitzenzentrum gibt es zwar einen Palliativdienst, die Abrechnung erfolgt jedoch nicht als palliativmedizinische Komplexbehandlung über den OPS-Schlüssel.

In 12 von 13 Zentren (92\%) wird der Dienst zu den Regelarbeitszeiten (wochentags 8-16 Uhr) angeboten.

Die Stellenbesetzung der im Palliativdienst beschäftigten Berufsgruppen zeigt innerhalb der CCCs deutliche Unterschiede. In allen 13 CCCs, die angeben, über einen Palliativdienst zu verfügen, ist der Palliativdienst ärztlich besetzt, in 11 Zentren ist zusätzlich eine konstante Besetzung durch Pflegekräfte gegeben. 7 Palliativdienste erfahren Unterstützung durch Fachpersonal aus der Psychologie. In 6 onkologischen Spitzenzentren gehört der Sozialdienst zum multiprofessionellen Team des Palliativdienstes. 2 Zentren verstärken ihre Multiprofessionalität durch die Physiotherapie. Kunsttherapeuten sind nur in 1 der CCCs dem Palliativdienst angehörig. 3 Zentren verstärken ihre Multiprofessionalität zusätzlich durch die Seelsorge, 2 zusätzlich durch die Zusammenarbeit mit einem Hospizdienst ( $\triangleright$ Abb. 1).

Beim Personalschlüssel reicht die Spannweite bei den Ärzten und den Pflegekräften jeweils von minimal 1 bis maximal 3 Vollzeitkräften. In der Berufsgruppe der Psychologen gibt es maximal 2 Vollzeitkräfte. Im Bereich der Sozialarbeit ist nur in 1 CCC das Palliativdienst-Team mit einer Vollzeitstelle ausgestattet. Die Physiotherapie ist maximal mit einer Halbtagsstelle vertreten, die Kunsttherapie mit einer Achtel-Stelle.

Eine Multiprofessionalität im Sinne der S3-Leitlinie für Palliativmedizin [2] war im Jahr 2016 somit insgesamt in 7 Palliativdiensten (54\%) gewährleistet. Folglich haben 7 CCCs (54\%) einen Palliativdienst, wie dieser in den S3-Leitlinien [2] und den BestPractice-Empfehlungen [6] beschrieben wird.

Insgesamt konnten eine Anzahl von 5844 (70,2\%) der erbrachten Leistungen der Dienste nicht erlösrelevant abgerechnet werden. 6 Zentren konnten durch die erbrachten palliativmedizinischen Leistungen keine Erlöse erzielen, in 7 Zentren war eine erlösrelevante Codierung möglich. Von diesen 7 Zentren konnten nur 2 Zentren mehr als 50 \% ihrer erbrachten Gesamtleistung für ein Zusatzentgelt kodieren (siehe $>$ Tab. 1). 
- Tab. 1 Anzahl der vom Palliativdienst betreuten Patienten sowie Anteil der erlösrelevant abrechenbaren Leistungen des Palliativdienstes.

\begin{tabular}{|c|c|c|c|}
\hline & $\begin{array}{l}\text { Anzahl erbrachter Leistungen/ } \\
\text { Anzahl der Patienten ohne } \\
\text { Vergütung }\end{array}$ & $\begin{array}{l}\text { Anzahl erbrachter Leistungen/ } \\
\text { Anzahl der Patienten mit } \\
\text { Vergütung }\end{array}$ & $\begin{array}{l}\text { Gesamtzahl erbrachter Leistungen/ } \\
\text { behandelter Patienten }\end{array}$ \\
\hline сcc. 1 & 269 & 350 & 619 \\
\hline ccc. 2 & 200 & 0 & 200 \\
\hline сcс.3 & 652 & 194 & 846 \\
\hline cсc. 4 & 464 & 0 & 464 \\
\hline ccc. 5 & 350 & 197 & 547 \\
\hline ccc.7 & 338 & 140 & 478 \\
\hline $\mathrm{ccc} .8$ & 464 & 0 & 464 \\
\hline ccc. 10 & 6 & 0 & 6 \\
\hline ccc. 11 & 171 & 0 & 171 \\
\hline ccc. 12 & 687 & 0 & 687 \\
\hline ccc. 13 & 864 & 249 & 1113 \\
\hline сcc.14 & 307 & 726 & 1033 \\
\hline ccc. 15 & 500 & 355 & 855 \\
\hline Summe & 5844 & 2480 & 8324 \\
\hline
\end{tabular}

\section{Anzahl palliativmedizinischer Komplexbehandlungen \\ im Jahr 2016 (ZE 60) \\ erbracht durch die Palliativdienste im CCC-Netzwerk}

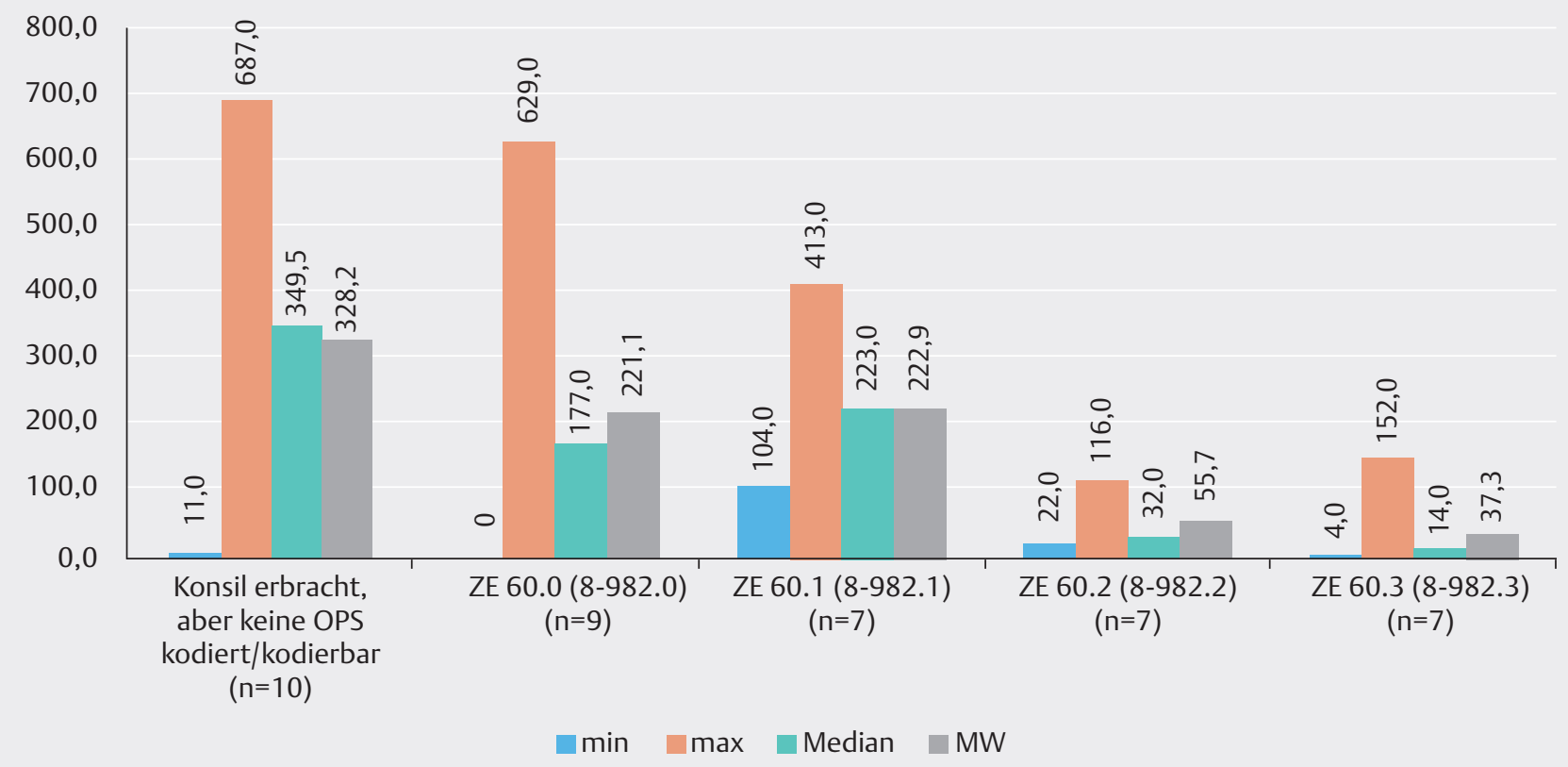

- Abb.2 Anzahl palliativmedizinischer Komplexbehandlungen im Jahr 2016 (ZE 60), erbracht durch die Palliativdienste im CCC-Netzwerk. CCC-Netzwerk = Netzwerk der Comprehensive Cancer Center; $\max =$ Maximum; $\min =$ Minimum; MW = Mittelwert; $\mathrm{n}=$ Anzahl der CCCs; OPS = Operationen- und Prozedurenschlüssel; ZE = Zusatzentgelt. 
- Abb. 2 zeigt, dass bei den palliativmedizinischen Komplexbehandlungen ein geringer Anteil der Leistungen OPS-relevant abrechenbar ist.

Auf die Frage, welche Abrechnungsmodalitäten für das Jahr 2017 geplant sind, gaben 5 Zentren an, nur noch nach dem neuen ZE 2017-133, 2 Zentren weiterhin nach ZE 60 und 3 Zentren nach beiden Abrechnungsformen abzurechnen zu wollen. 3 Zentren machten bei dieser Frage keine Angabe.

Bezüglich der Verhandlungen zum neuen Zusatzentgelt ZE 133 gaben 2 CCCs an, dass die Verhandlungen diesbezüglich bereits abgeschlossen sind. Davon waren in 1 Zentrum die Verhandlungen mit den Kostenträgern bereits 1-malig gescheitert und mit 587 Euro pro Leistungserbringung bzw. Fall, unabhängig von dem zeitlichen Aufwand des Falles, abgeschlossen. Das andere CCC konnte sich bei den abgeschlossenen Verhandlungen kein Zusatzentgelt sichern; es wird nach einer nicht kalkulierten Vergütung abgerechnet. In 10 CCCs wurde zum Zeitpunkt der Befragung noch kein Abschluss der Verhandlungen für das Zusatzentgelt ZE 133 genannt. Dabei berichtete 1 CCC, dass mit einer Fallpauschale in Höhe von 600 Euro abgerechnet wird. Ein anderes CCC betonte, dass von Mitarbeitern des Controllings für 2017 noch das Zusatzentgelt ZE 60 bevorzugt wird.

\section{Diskussion}

Erstmals wird die Anzahl und Situation von Palliativdiensten in den deutschen onkologischen Spitzenzentren dargestellt. In fast allen Spitzenzentren wird ein Palliativdienst angeboten, jedoch bzgl. der beteiligten Professionen, Stellenumfang und Finanzierungsarten sehr heterogen und nur zum Teil den Kriterien der nationalen Leitlinien entsprechend. Die Ergebnisse zeigen einen großen Nachholbedarf und können als Vergleich für zukünftige Erhebungen zur Weiterentwicklungsbeschreibung nach Einführung der neuen OPS und ZE dienen.

Als Bestandteil der Versorgungsstruktur in einem Krankenhaus wird ein palliativmedizinischer Dienst sowohl in der deutschen S3Leitlinie für Palliativmedizin [2] als auch in den Best-Practice-Empfehlungen zur Integration der Palliativmedizin in ein von der Deutschen Krebshilfe gefördertes CCC [6] gefordert. Palliativdienste, wie sie in Deutschland bezeichnet werden, werden beispielsweise in Österreich und in der Schweiz unter dem Begriff Palliativkonsiliardienst geführt [14-16]. In anderen, insbesondere englischsprachigen Ländern werden Begriffe wie Palliative Care Team, Inpatient Palliative Care Consult Service, Specialized Inpatient Palliative Care Service, Hospital Support Team, Hospital Supportive Care Team oder auch Hospital Mobile Team verwendet [1619]. Die divergierende Bezeichnung ist vermutlich unter anderem der Teamzusammensetzung, dem definierten Auftragsziel und der unterschiedlichen Leistungsvergütung in den jeweiligen Ländern geschuldet.

Das multiprofessionelle Team eines Palliativdienstes in einem CCC in Deutschland sollte neben Ärzten und Pflegekräften mindestens 1 Person aus einer weiteren Berufsgruppe, beispielsweise aus der Physiotherapie oder aus dem Bereich Sozialarbeit, umfassen $[2,6]$. Im Gegensatz zu Deutschland besteht die personelle Mindestanforderung an einen Palliativdienst in der Schweiz aus
1 ärztlichen Mitarbeiter und 1 Person aus dem Pflegebereich [15]. Das Team des Palliativkonsiliardienstes in Österreich ist durch ein „Stammpersonal“ aus Ärzten, Pflegefachkräften und Sozialarbeitern definiert $[14,20] .13$ von 15 deutschen onkologischen Spitzenzentren, die an der Befragung teilgenommen haben, haben die Frage, über einen Palliativdienst zu verfügen, unabhängig davon, ob die Kriterien der S3-Leitlinie und der Best-PracticeEmpfehlungen erfüllt werden, bejaht. Nur 7 Palliativdienste der deutschen CCCs erfüllen jedoch das Kriterium der Multiprofessionalität. Eine vergleichbare Situation hinsichtlich der Personalaufstellung gibt es auch in anderen Ländern, wie beispielsweise in der Schweiz. Dort besteht die personelle Mindestanforderung an einen Palliativdienst aus 1 ärztlichen Mitarbeiter und 1 Person aus dem Pflegebereich [15].

Hinsichtlich der personellen Mindestbesetzung gibt es in Österreich für einen Palliativkonsiliardienst genaue Vorschriften. Pro 150 bis 250 Betten werden 2 Vollzeitäquivalente und 1 zusätzliches Vollzeitkräfteäquivalent je weiterer 250 Betten in der Gruppe des „Stammpersonals“ aus Ärzten, Pflegefachkräften und Sozialarbeitern gefordert [14]. In Deutschland liegen diesbezüglich bislang keine verbindlichen Angaben vor. Im Rahmen der Befragung variieren die Angaben zur Personalstärke bei den Ärzten und den Pflegekräften jeweils von minimal 1 bis maximal 3 Vollzeitkräfteäquivalenten. Die Personalstärke in allen weiteren Berufsgruppen wird in allen Standorten der CCCs, die laut Angabe über einen Palliativdienst verfügen, ausschließlich mit Teilzeitstellen angegeben.

Gemäß der Regelung vor dem Jahr 2017 wird die sogenannte „palliativmedizinische Komplexbehandlung“ über den OPS-Code 8-982 nach ZE 60 abgerechnet. Seit Januar 2017 erfolgt über den OPS-Code 8-98h die Leistungsabrechnung nach ZE 2017-133 bzw. 2017-134, die zwischen einer „spezialisierten palliativmedizinischen Komplexbehandlung durch einen internen Palliativdienst“ und einer „spezialisierten palliativmedizinischen Komplexbehandlung durch einen externen Palliativdienst" unterscheidet [12]. Im Gegensatz zum ZE 60, über den die erbrachten Leistungen nach Tagen abgerechnet werden, kann über den ZE 2017-133 bzw. -134 eine stundengenaue Abrechnung erfolgen.

Im Rahmen der Befragung konnte eruiert werden, dass rund $70 \%$ der erbrachten Leistungen der Palliativdienste nach ZE 60 nicht vergütet werden können, sei es, weil die Behandlungstage unter 7 Tagen lagen oder weil keine OPS im Sinne einer palliativmedizinischen Komplexbehandlung nach 8-982 kodierbar war. Während in 7 Zentren eine erlösrelevante Codierung möglich war, konnten in 6 Zentren keine Zusatzerlöse gewonnen werden. Nur 2 von 7 Zentren konnten mehr als $50 \%$ ihrer erbrachten Gesamtleistung für ein Zusatzentgelt kodieren (siehe $>$ Tab. 1). Bei den palliativmedizinischen Komplexbehandlungen ist nur ein geringer Anteil der Leistungen OPS-relevant abrechenbar ( $\triangleright$ Abb. 2). Dies könnte daran liegen, dass der OPS-Code 8-982 bestimmte Mindestmerkmale erfordert, die nicht vollständig erfüllt werden konnten. Der zeitliche Aufwand von insgesamt mindestens 6 Stunden pro Patient und vollständiger Woche patientenbezogen, d. h. persönlicher Kontakt mit Patient und/oder Angehörigen ist beispielsweise eine Voraussetzung. Dabei können diese Gespräche von allen Berufsgruppen durchgeführt werden [12]. Die nur eingeschränkten Erlösmöglichkeiten für einen Pallia- 
tivdienst erschweren die Umsetzungen der Empfehlungen. Inwiefern der Code 8-98h ab 2017 durch die veränderte zeitliche Staffelung bei gleichzeitig höheren Qualitätsmerkmalen zu mehr abrechenbare Fällen führen wird, bleibt abzuwarten. In unserer Befragung bezüglich der geplanten Abrechnungsmodalitäten für das Jahr 2017 beabsichtigten 5 Zentren, nach der neuen ZE 2017-133, 2 Zentren weiterhin nach ZE 60 und 3 Zentren nach beiden Abrechnungsformen abzurechnen. Die verbleibenden Zentren waren diesbezüglich noch unentschieden. Bislang wird die Abrechnung der Palliativdienste lokal unterschiedlich gehandhabt, je nach Verhandlung ist der Erlös unterschiedlich.

Per Definition [2, 6] ist es nicht Aufgabe eines Palliativdienstes, ambulante Patienten zu betreuen. In der Abrechnung werden nur stationär geführte Patienten berücksichtigt. Bezugnehmend auf die frühe Integration („Early Integration“) und auf die Best-Practice-Empfehlungen [6] soll Patienten nach der Diagnose einer nicht heilbaren Krebserkrankung Palliativversorgung angeboten werden, unabhängig von der Durchführung einer tumorspezifischen Therapie. Arbeiten von May et al. [21-23] haben gezeigt, dass eine frühzeitige Palliativversorgung mit deutlich höheren Kosteneinsparungen verbunden ist. Aus wirtschaftlicher Sicht könnten damit durch eine frühe Integration in die Palliativmedizin Kosten eingespart werden, was auch eine erlösrelevante Vergütung ambulant geführter Patienten durch den Palliativdienst rechtfertigen würde. Neben den wirtschaftlichen Aspekten hat die frühe Integration in die Palliativmedizin vor allem auch positive Auswirkungen auf patientenrelevante Endpunkte wie Lebensqualität und Lebenszeit [24-28].

Letztendlich zeigt sich durch die vorliegende Erhebung eine große Heterogenität hinsichtlich des Angebots und der Abrechnungsmodalitäten von Palliativdiensten. Zum anderen gibt es bisher keine zufriedenstellende, kongruente Einigung zur Abrechnung der von den Palliativdiensten erbrachten Leistungen in Deutschland. Die neue Komplexpauschale hat dazu geführt, dass definierte Bedingungen geschaffen wurden, um entsprechende von den Palliativdiensten erbrachte Leistungen auch erlösrelevant anbieten zu können. Es hat überdies stärker ins Bewusstsein gebracht, dass die spezialisierte Palliativversorgung nicht „en passant“ durch dafür nicht qualifiziertes Personal als erlössteigernde Mitnahmeeffekte erbracht werden kann. Die Zentren, die eine adäquate und multiprofessionelle Personalausstattung in der spezialisierten Palliativmedizin aufweisen, können die Leistungen des Palliativdienstes in der Regel auch erlösrelevant abrechnen. Dies sollte neben den Empfehlungen der S3-Leitlinie Palliativmedizin und der Best-Practice-Empfehlung der Deutschen Krebshilfe als Motivation dienen, diese Dienste nicht nur in den deutschen onkologischen Spitzenzentren multiprofessionell und personell adäquat auszustatten.

\section{Limitationen}

Bei der Untersuchung handelt es sich um eine Erhebung der Ist-Situation der Onkologischen Spitzenzentren (CCCS) in Deutschland und damit ausschließlich um Kliniken der Maximalversorgung im Sinne einer Stichprobenbefragung. Der Status anderer Kliniken der Grundund Schwerpunktversorgung kommt dabei nicht zur Darstellung.
Bislang gibt es ausschließlich für Bayern Daten zur Anzahl an Palliativdiensten [29]. In Deutschland ist die Anzahl bestehender Palliativdienste nicht bekannt. Weiterführende Untersuchungen könnten neue Erkenntnisse schaffen.

\section{KERNAUSSAGEN}

- Palliativdienste sind in den deutschen CCCs regelhaft vorhanden, erfüllen jedoch nur zu ca. 50 \% die zur Abrechnung geforderte Bedingung der Multiprofessionalität.

- Im Jahr 2017 wurden stundengenau abrechenbare Zusatzentgelte neu eingeführt, welche Verbesserungen schaffen und zur Kostendeckung beitragen könnten.

- Bisher sind notwendige und sinnvolle Leistungen entgangen, welche mit einer Behandlungsdauer $<7$ Tagen nach neuem ZE abgerechnet werden können.

\begin{tabular}{|c|c|}
\hline \multicolumn{2}{|c|}{ GLOSSAR/ABKÜRZUNGSVERZEICHNIS } \\
\hline AG Palliativmedizin & Arbeitsgruppe für Palliativmedizin \\
\hline a.m. & vor Mittag (ante meridiem) \\
\hline CCC & Comprehensive Cancer Center \\
\hline CCC-Netzwerk & $\begin{array}{l}\text { Netzwerk der Comprehensive Cancer } \\
\text { Center }\end{array}$ \\
\hline DKH & Deutsche Krebshilfe \\
\hline InEK & $\begin{array}{l}\text { Institut für das Entgeltsystem im } \\
\text { Krankenhaus }\end{array}$ \\
\hline $\max$ & Maximum \\
\hline $\min$ & Minimum \\
\hline MW & Mittelwert \\
\hline $\mathrm{n}$ & Anzahl \\
\hline OPS & Operationen- und Prozedurenschlüssel \\
\hline p.m. & nach Mittag (post meridiem) \\
\hline ZE & Zusatzentgelt \\
\hline
\end{tabular}

Interessenkonflikt

Die Zentren, in denen die Autoren angestellt sind, werden von der Deutschen Krebshilfe gefördert. Weitere Interessenskonflikte in Bezug auf das Manuskript bestehen nicht.

Danksagung

Wir danken der Deutschen Krebshilfe e. V. für die Unterstützung des Kooperationsprojekts der AG Palliativmedizin im CCC-Netzwerk. Unser Dank gilt auch den CCC-Direktoren und ihren Vertretern für die entgegenkommende Teilnahme an der Befragung.

Die vorliegende Arbeit ist Teil der Promotionsleistung von Sarah Lödel und wurde in Erfüllung der Anforderungen für den Erhalt des Doktortitels Dr. rer. biol. hum. durchgeführt. 
[1] Deutsche Gesellschaft für Palliativmedizin. Wegweiser Hospiz- und Palliativversorgung Deutschland. Im Internet: www.wegweiser-hospiz-pal liativmedizinde/de/angebote/erwachsene/12-palliativdienste_im_kran kenhaus; Stand: 12.06.2019

[2] Leitlinienprogramm Onkologie (Deutsche Krebsgesellschaft, Deutsche Krebshilfe, AWMF): Palliativmedizin für Patienten mit einer nicht heilbaren Krebserkrankung, Langversion 2.01 (Konsultationsfassung), 2019, AWMF-Registernummer: 128/001OL. Im Internet: www.leitlinienpro gramm-onkologie.de/leitlinien/palliativmedizin/; Stand: 12.06.2019

[3] Müller-Busch H. Definitionen und Ziele in der Palliativmedizin. Internist 2011; 52: 7-14. doi:10.1007/s00108-010-2688-0

[4] Jack B, Hillier V, Williams A et al. Improving cancer patients' pain: the impact of the hospital specialist palliative care team. Eur J Cancer Care 2006; 15: 476-480. doi:10.1111/j.1365-2354.2006.00690.x

[5] Hui D, Elsayem A, De la Cruz M et al. Availability and integration of palliative care at US cancer centers. Jama 2010; 303: 1054-1061. doi:10.1001/jama.2010.258

[6] Arbeitsgruppe Palliativmedizin im CCC-Netzwerk. Best Practice- Empfehlungen zur Integration der Palliativmedizin in ein von der Deutschen Krebshilfe gefördertes Comprehensive Cancer Center (CCC) (Dezember 2017). Im Internet: www.ccc-netzwerk.de/fileadmin/Inhalte/Downlo ads/PDF/Best_Practice_Handreichung.pdf; Stand: 12.06.2019

[7] Grudzen CR, Richardson LD, Hopper SS et al. Does palliative care have a future in the emergency department? Discussions with attending emergency physicians. J Pain Symptom Manage 2012; 43: 1-9. doi:10.1016/j.jpainsymman.2011.03.022

[8] Enguidanos S, Housen P, Goldstein R et al. Physician and nurse perceptions of a new inpatient palliative care consultation project: implications for education and training. J Palliat Med 2009; 12: 1137-1142. doi:10.1089/jpm.2009.0131

[9] Hamilton DG. Believing in patients' beliefs: physician attunement to the spiritual dimension as a positive factor in patient healing and health. Am J Hosp Palliat Care 1998; 15: 276-279. doi:10.1177| 104990919801500509

[10] Maddison AR, Malik S, Smaggus A. Inpatient Palliative Care Consultations From a Canadian Clinical Teaching Unit: Who is Referred and When? J Palliat Care 2018; 33 (4): 204-208. doi:10.1177| 0825859718781363

[11] Smith C, Bosanquet N, Riley J et al. Loss, transition and trust: perspectives of terminally ill patients and their oncologists when transferring care from the hospital into the community at the end of life. BMJ Support Palliat Care 2016. doi:10.1136/bmjspcare-2015-001075

[12] Deutsche Krankenhausgesellschaft (DKG), GKV-Spitzenverband, Verband der privaten Krankenversicherung (PKV), Institut für das Entgeltsystem im Krankenhaus (InEK GmbH). Deutsche Kodierrichtlinien. Allgemeine und Spezielle Kodierrichtlinien für die Verschlüsselung von Krankheiten und Prozeduren (Version 2019). Im Internet: www.dkgev. de/fileadmin/default/Mediapool/2_Themen/2.4._Medizin_und_Wissen schaft/2.4.2._Medizinische_Klassifikationen/2.4.2.3._Kodierrichtlinien/ DKR_2019.pdf; Stand: 12.06.2019

[13] Stevens S, Kloke M. Palliativkomplexbehandlung in der Onkologie. Onkologe 2013; 19: 408-414. doi:10.1007/s00761-013-2445-9

[14] Österreichisches Bundesinstitut für Gesundheitswesen, Budesministerium für Gesundheit. Abgestufte Hospiz-und Palliativversorgung für Erwachsene (Aktualisierung 2014). Im Internet: www.sozialministerium. at/cms/site/attachments/3/6/7/CH3967/CMS1103710970340/bro schuere_hospiz-_und_palliativversorgung_1_12_2014.pdf; Stand: 12.06.2019

[15] Bundesamt für Gesundheit (BAG), palliative ch und Schweizerische Konferenz der kantonalen Gesundheitsdirektorinnen und -direktoren (GDK). Versorgungsstrukturen für spezialisierte Palliative Care in der Schweiz (Dezember 2014). BAG-Publikationsnummer: GP 08.12 2000d 800f 30EXT1209. Im Internet: www.palliative.ch/fileadmin/user_upload/ palliative/publikum/2_PalliativeCare/versstrukt-spez-pc-ch.pdf; Stand: 12.06.2019

[16] Radbruch L, Payne S. Standards und Richtlinien für Hospiz-und Palliativversorgung in Europa: Teil 2. Zeitschrift für Palliativmedizin 2011; 12: 260-270. doi:10.1055/s-0031-1276957

[17] Atayee RS, Sam AM, Edmonds KP. Patterns of Palliative Care Pharmacist Interventions and Outcomes as Part of Inpatient Palliative Care Consult Service. J Palliat Med 2018; 21: 1761-1767. doi:10.1089/jpm.2018. 0093

[18] Arias-Casais N, Garralda E, Rhee JY et al. EAPC Atlas of Palliative Carein Europe 2019. Vilvoorde: EAPC Press; 2019. Im Internet: www. eapcnet. wordpress.com/2019/05/24/new-edition-of-eapc-atlas-of-palliativecare-in-europe-launches-at-16th-eapc-world-congress-in-berlin/; Stand: 12.06.2019

[19] Kotzsch F, Stiel S, Heckel M et al. Care trajectories and survival after discharge from specialized inpatient palliative care-results from an observational follow-up study. Support Care Cancer 2015; 23: 627-634. doi:10.1007/s00520-014-2393-y

[20] Uritsky T], Atayee RS, Herndon CM et al. Ten Tips Palliative Care Pharmacists Want the Palliative Care Team to Know When Caring for Patients. J Palliat Med 2018; 21: 1017-1023. doi:10.1089/jpm.2018.0187

[21] Watzke H. Aus der Österreichischen Palliativgesellschaft-Prozesshandbuch für Hospiz-und Palliativeinrichtungen. Zeitschrift für Palliativmedizin 2013; 14: 6. doi:10.1055/s-0032-1319099

[22] Grießmeier B, Krauß O, Roschmann R et al. Curio im Auftrag der Bundesarbeitsgemeinschaft Psychosoziale Versorgung im Akutkrankenhaus. Leitfaden zum OPS 2018, Psychosoziale Leistungen im somatischen Akutkrankenhaus dokumentieren und kodieren (14. Version: 01.01.2018). Im Internet: www.bdp-klinische-psychologie.de/service/ downloads/Kodierleitfaden_BAG_PVA_2018.pdf; Stand: 12.06.2019

[23] May P, Garrido MM, Cassel JB et al. Prospective Cohort Study of Hospital Palliative Care Teams for Inpatients With Advanced Cancer: Earlier Consultation Is Associated With Larger Cost-Saving Effect. J Clin Oncol 2015; 33: 2745-2752. doi:10.1200/jco.2014.60.2334

[24] May P, Garrido MM, Cassel JB et al. Palliative care teams' cost-saving effect is larger for cancer patients with higher numbers of comorbidities. Health Aff 2016; 35: 44-53. doi:10.1377/hlthaff.2015.0752

[25] Temel JS, Greer JA, Muzikansky A et al. Early palliative care for patients with metastatic non-small-cell lung cancer. N Engl J Med 2010; 363 : 733-742. doi:10.1056/NEJMoa1000678

[26] Yoong J, Park ER, Greer JA et al. Early palliative care in advanced lung cancer: a qualitative study. JAMA Intern Med 2013; 173: 283-290. doi:10.1001/jamainternmed.2013.1874

[27] Otsuka M, Koyama A, Matsuoka $\mathrm{H}$ et al. Early palliative intervention for patients with advanced cancer. Jpn J Clin Oncol 2013; 43: 788-794. doi:10.1093/jjco/hyt074

[28] Irwin KE, Greer JA, Khatib J et al. Early palliative care and metastatic nonsmall cell lung cancer: potential mechanisms of prolonged survival. Chron Respir Dis 2013; 10: 35-47. doi:10.1177/1479972312471549

[29] Ferrell B, Sun V, Hurria A et al. Interdisciplinary Palliative Care for Patients With Lung Cancer. J Pain Symptom Manage 2015; 50: 758-767. doi:10.1016/j.jpainsymman.2015.07.005

[30] Anneser J, Thurn T, Borasio GD. Entwicklung der Palliativmedizinischen Dienste (PMD) in Bayern 2011-2015: Aufgaben, Akzeptanz, Struktur und Finanzierung. Gesundheitswesen 2018; 80: 888-893. doi:10.1055/ s-0043-103950 